Supplement of Geosci. Model Dev., 10, 2547-2566, 2017

https://doi.org/10.5194/gmd-10-2547-2017-supplement

(C) Author(s) 2017. This work is distributed under

the Creative Commons Attribution 3.0 License.

(c) (1)

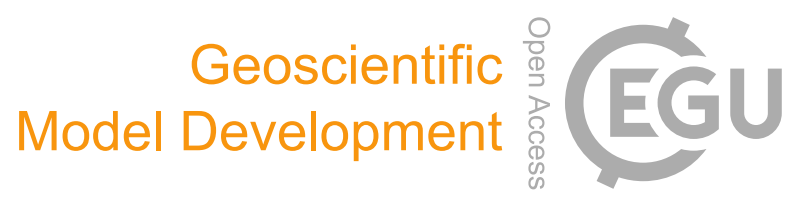

Supplement of

\title{
A multi-diagnostic approach to cloud evaluation
}

Keith D. Williams and Alejandro Bodas-Salcedo

Correspondence to: Keith Williams (keith.williams@metoffice.gov.uk)

The copyright of individual parts of the supplement might differ from the CC BY 3.0 License. 


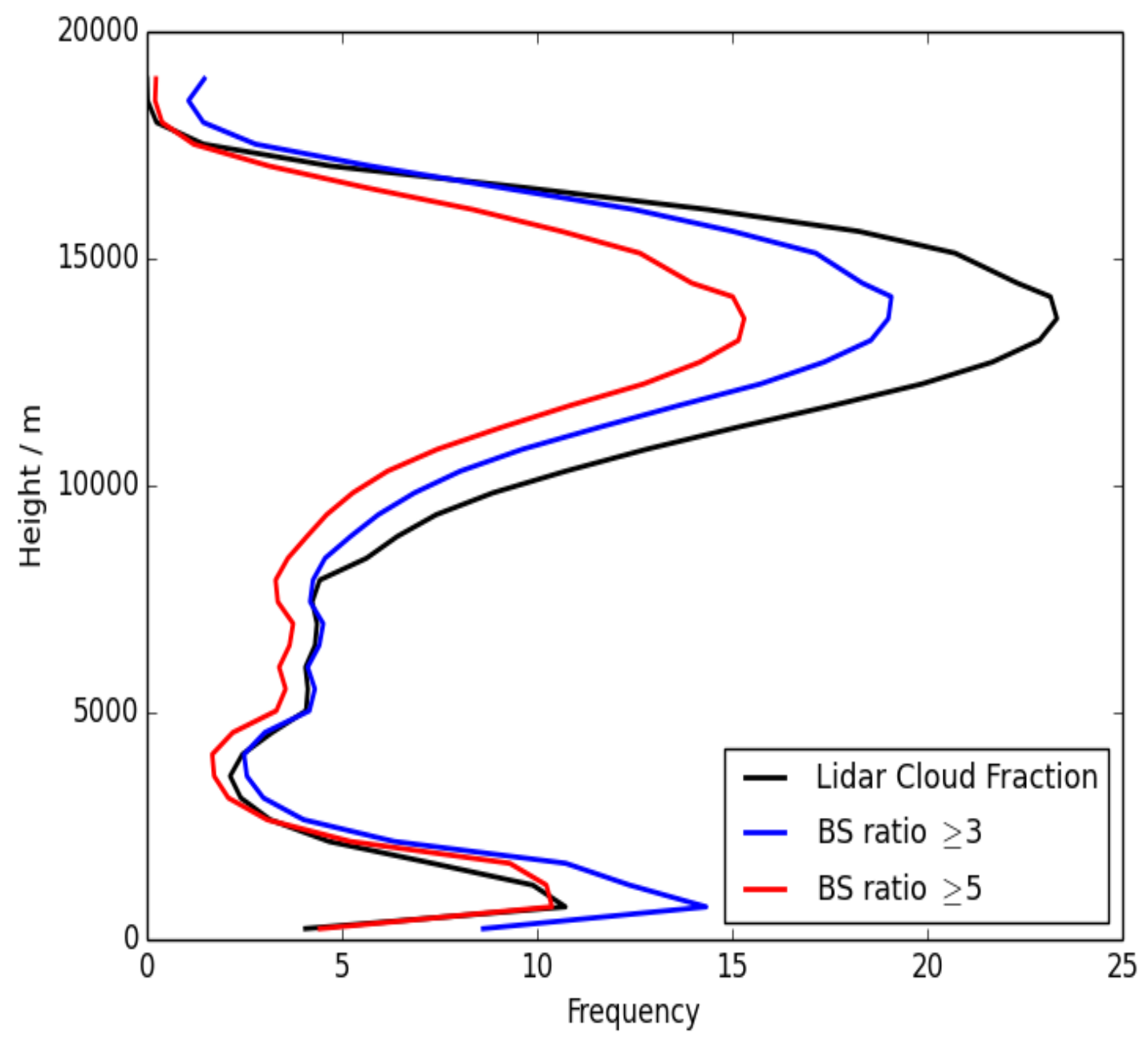

Figure S1: CALIPSO observed height-cloud frequency histograms for the tropics $\left(20^{\circ} \mathrm{N}-20^{\circ} \mathrm{S}\right)$. Black is the lidar cloud fraction obtained from the RL-GEOPROF product (and thus is based on the lidar cloud detection algorithm). Blue and red are obtained from CALIPSO heightbackscatter ratio histograms by adding together the backscatter ratio bins $\geq 3$ and $\geq 5$ respectively. 
GA6

NH DJF
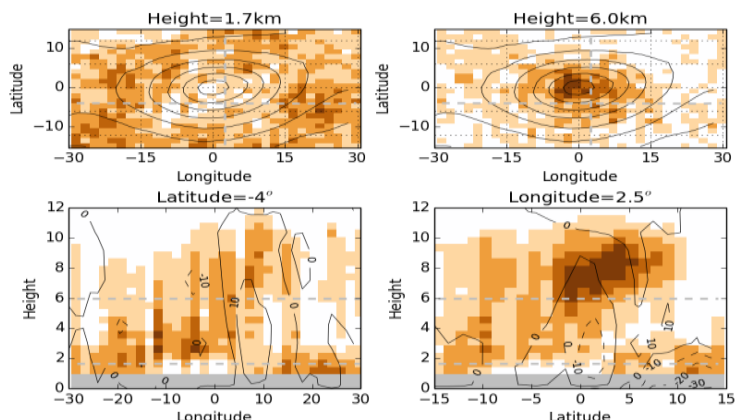

Longitude
Longitude $=2.5$

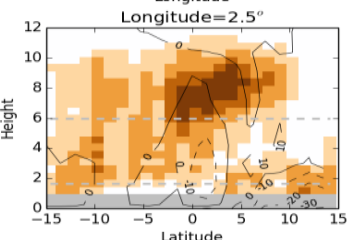

\begin{tabular}{lllllll} 
Longitude & & & \multicolumn{1}{c}{ Latitude } \\
\hline$-0.25-0.20-0.15-0.10-0.05$ & 0.05 & 0.10 & 0.15 & 0.20 & 0.25
\end{tabular}

NH JJA
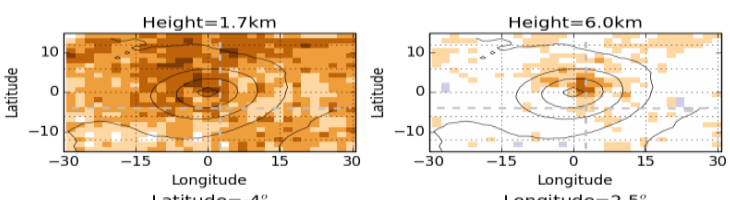

Longitude
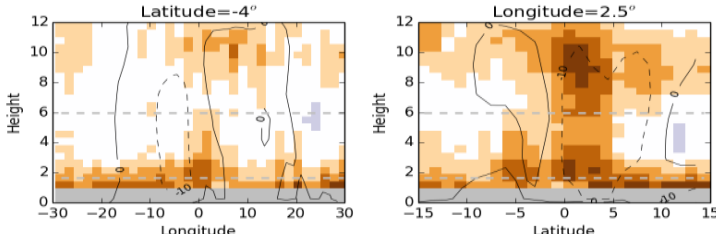

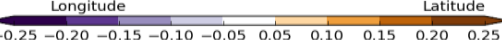

SH JJA
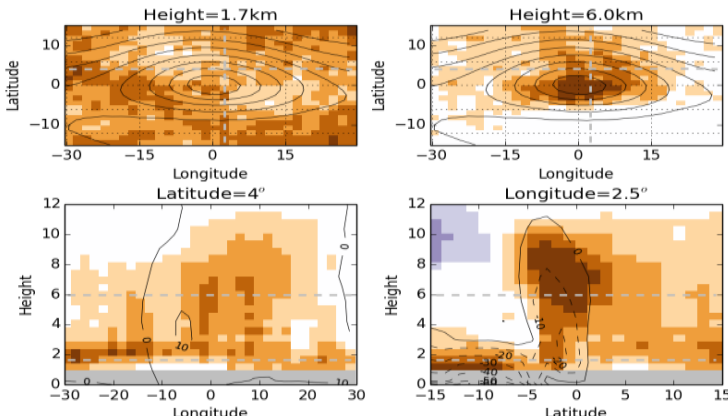

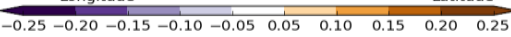

SH DJF
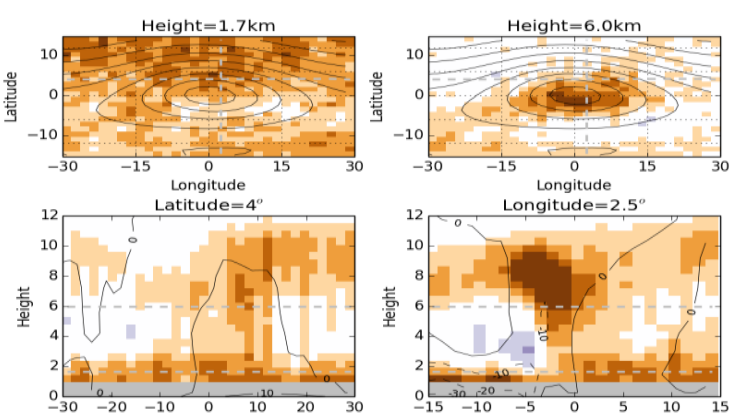

angitude $=25$

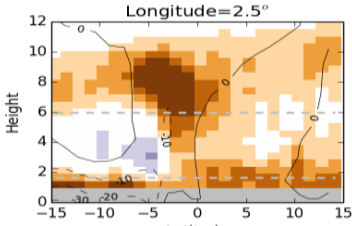

$0.25-0.20-0.15-0.10-0.050 .05010 \quad 0.150 .200 .25$
GA7

NH DJF
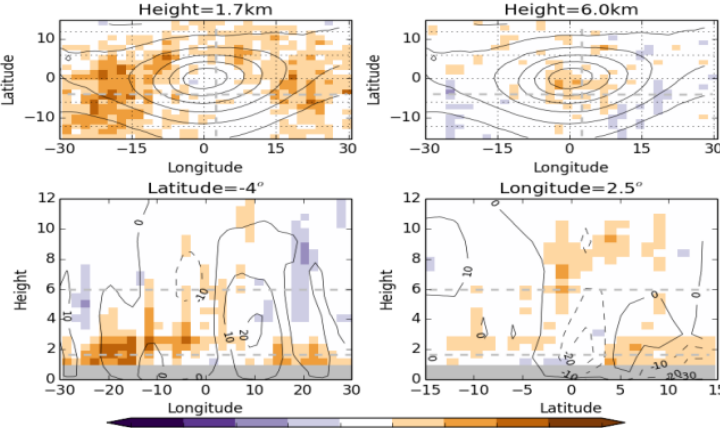

NH JJA
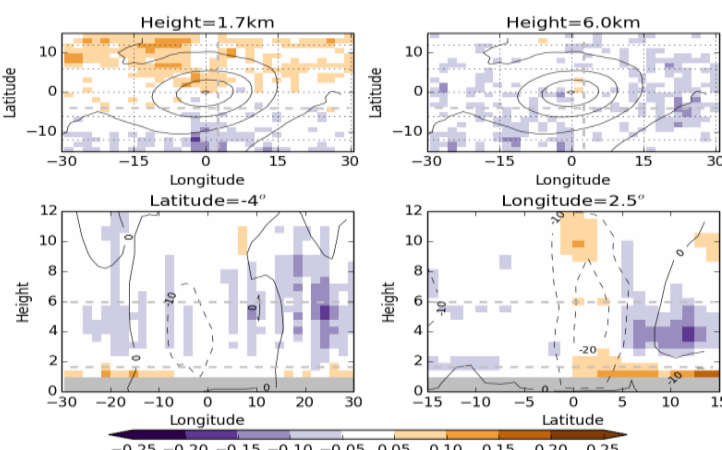

SH JJA
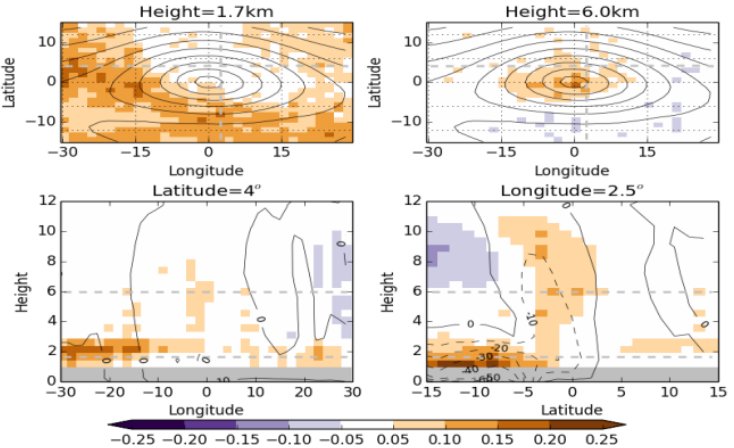

SH DJF
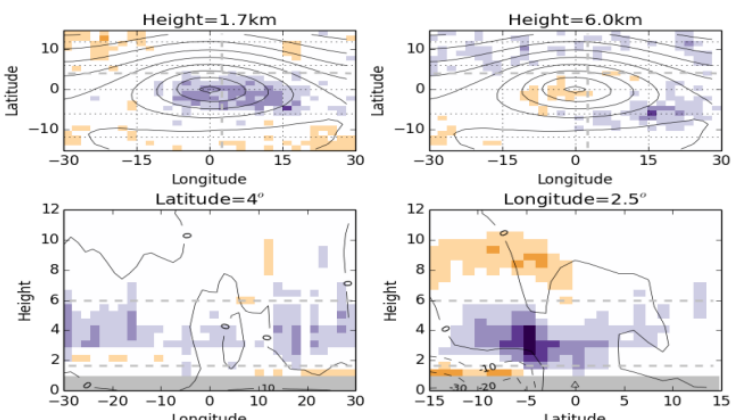

$\begin{array}{lllllll}0.25-0.20-0.15-0.10-0.05 & 0.05 & 0.10 & 0.15 & 0.20 & 0.25\end{array}$

Figure S2: Cloud fraction bias for GA6 (left) and GA7 (right) composite cyclones (as per Figure 7 in main paper). From top to bottom are northern hemisphere winter, northern hemisphere summer, southern hemisphere winter and southern hemisphere summer. 
GA6

NH DJF
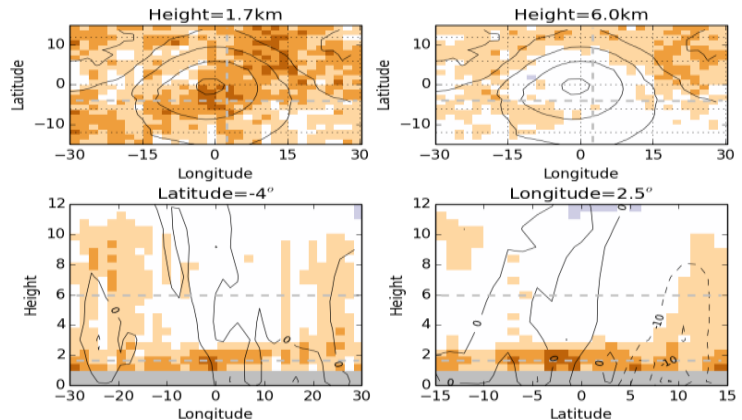

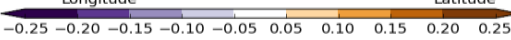

NH JJA
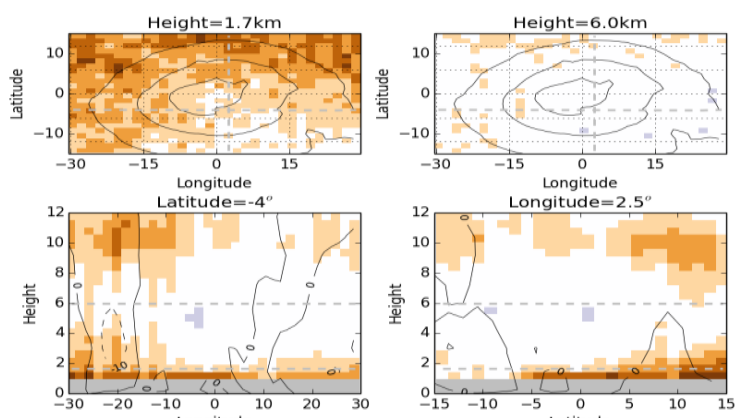

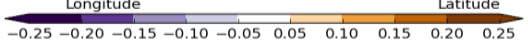

SH JJA
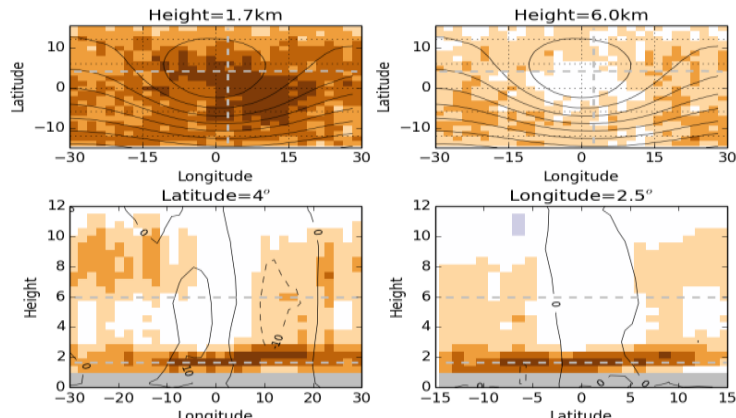

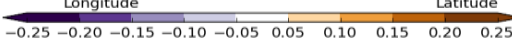

SH DJF
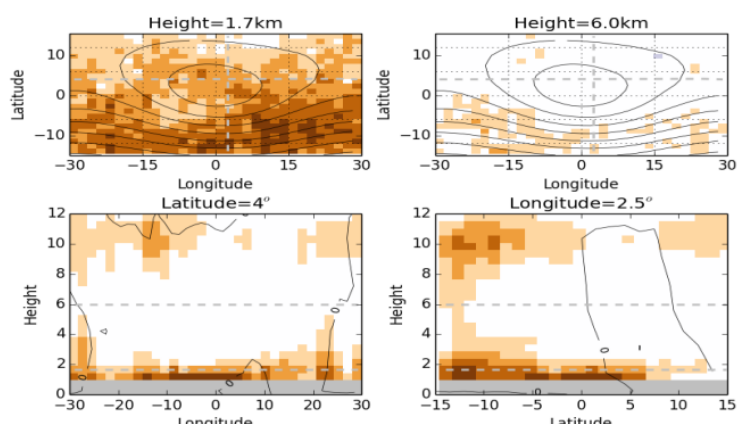

$\begin{array}{llllll}-0.25-0.20-0.15-0.10-0.05 & 0.05 & 0.10 & 0.15 & 0.20 & 0.25\end{array}$
GA7

NH DJF
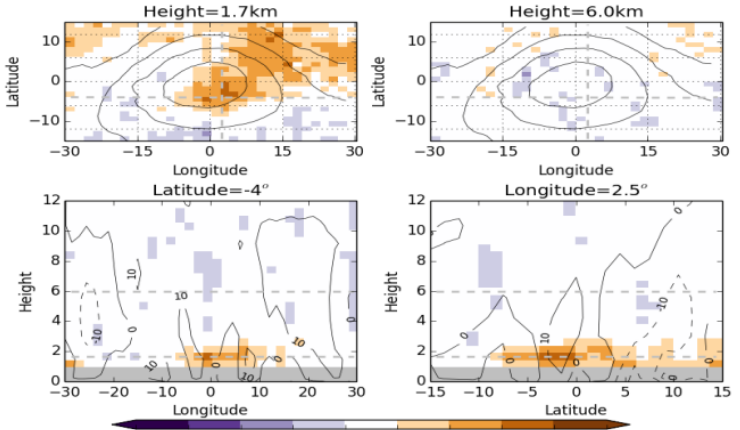

NH JJA
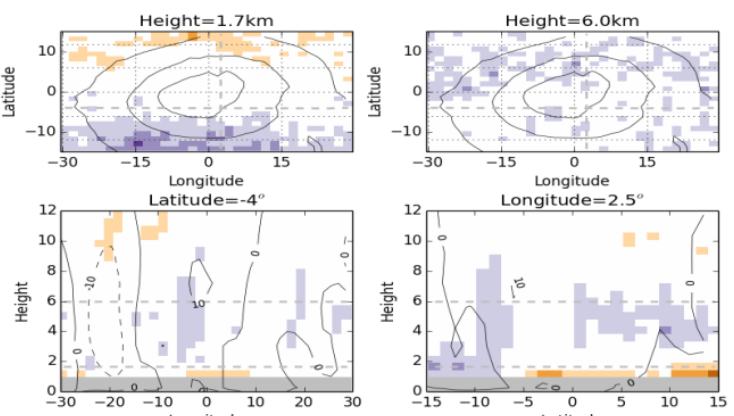

\begin{tabular}{llllll} 
& & & & Latitude \\
\hline$-0.25-0.20-0.15-0.10-0.05$ & 0.05 & 0.10 & 0.15 & 0.20 & 0.25
\end{tabular}

SH JJA
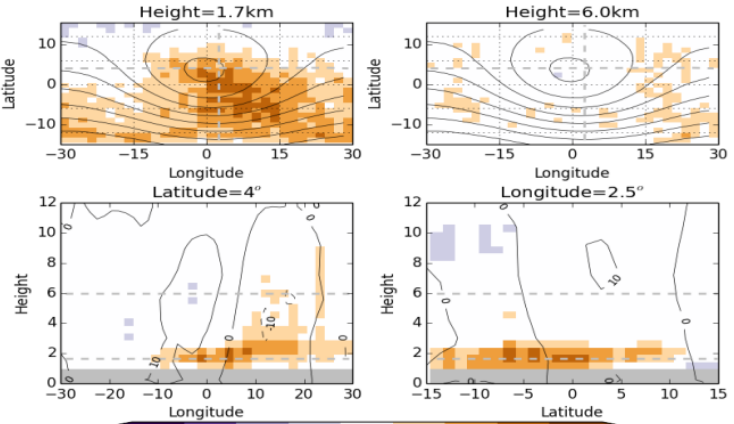

SH DJF
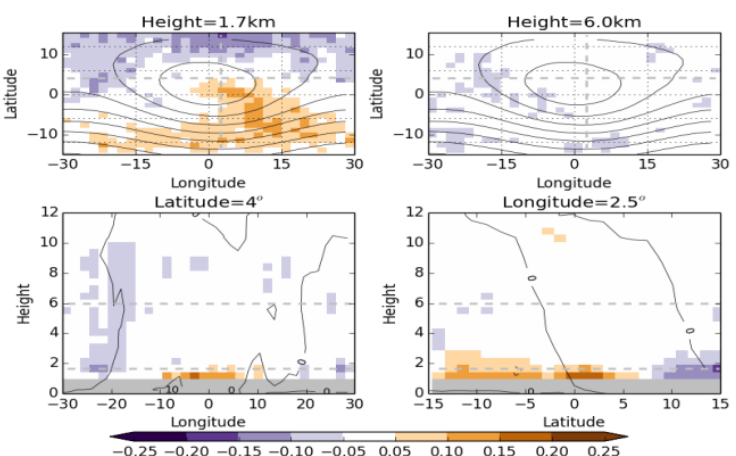

Figure S3: As Supplementary figure 2 but for anticyclones. 
NH DJF

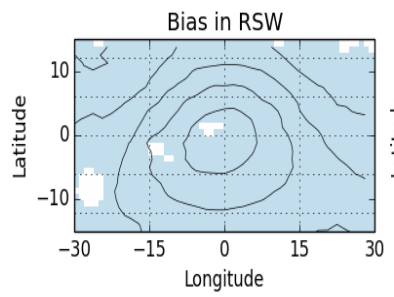

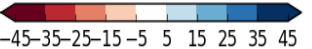

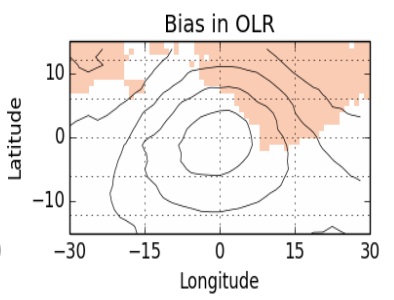

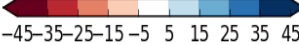

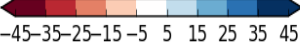

SH JJA

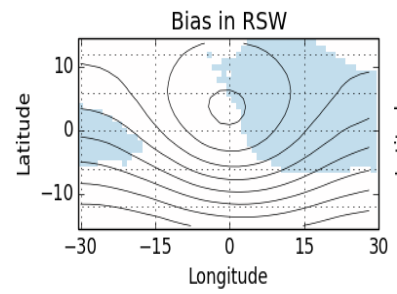

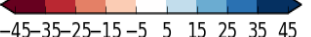

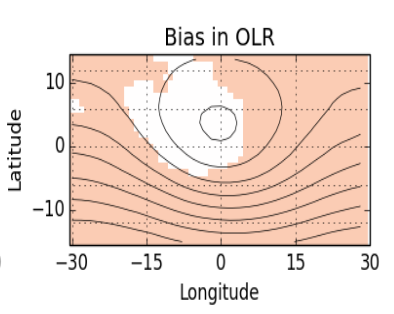

\begin{tabular}{llllll}
\hline & 1 & 1 & & \\
$-45-35-25-15-5$ & 5 & 15 & 25 & 35 & 45
\end{tabular}
NH JJA
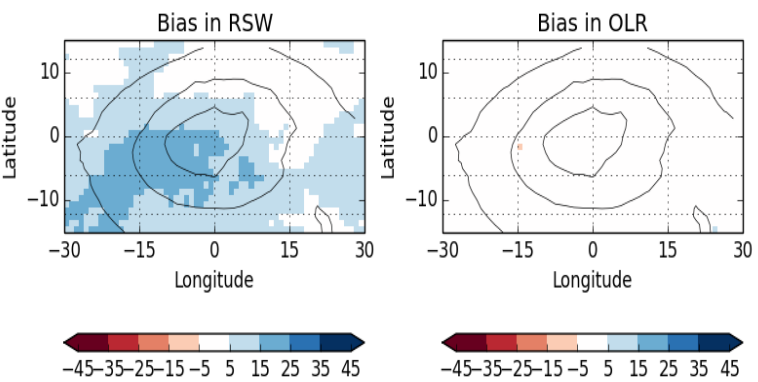

\section{SH DJF}
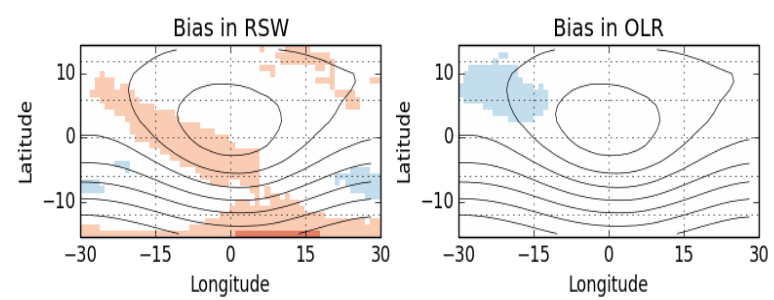

$-45-35-25-15-5 \quad 5 \quad 15 \quad 253545$

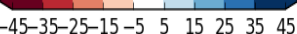

Figure S4: RSW and OLR bias for GA7 composite anticyclones (as per Figure 9 in main paper). 